\title{
On the Relations between Lucas Sequence and Fibonacci-like Sequence by Matrix Methods
}

\author{
Arfat Ahmad Wani ${ }^{\mathrm{a}}$ and V. H. Badshah ${ }^{\mathrm{b}}$ \\ ${ }^{a, b}$ School of Studies in Mathematics, Vikram University Ujjain, India.
}

Received: 21 February 2017; Accepted: 08 June 2017; Published: 08 November 2017

\begin{abstract}
In the present paper first and foremost we introduce a generalization of a classical Fibonacci sequence which is called a Fibonacci-Like sequence and at hindmost we obtain some relationships between Lucas sequence and Fibonacci-Like sequence by using two cross two matrix representation to the Fibonacci-Like sequence. The most worth noticing cause of this article is our proof method, since all the identities are proved by using matrix methods.
\end{abstract}

Index Terms: Fibonacci Sequence, Lucas Sequence, Generalization of Fibonacci Sequence and Matrix Method.

Mathematics Subject Classification: 11B37, 11B39, 40C05.

(C) 2017 Published by MECS Publisher. Selection and/or peer review under responsibility of the Research Association of Modern Education and Computer Science

\section{Introduction}

Fibonacci numbers have many applications as well as interesting properties almost in every field of science such as in Physics, Biology, Computer Science, Engineering, Mathematics (Algebra, Geometry and Number Theory itself). Furthermore Fibonacci and Lucas numbers have long interested mathematicians for their intrinsic theory and applications. Fibonacci numbers and Lucas numbers continue to provide invaluable opportunities for exploration, and contribute handsomely to the beauty of mathematics, especially number theory, one can see the citations $[9,10,13]$.

The Fibonacci and Lucas sequences are defined by the recurrence relations:

\footnotetext{
* Corresponding author. Tel.: +919797029821

E-mail address: atharaslam3@gmail.com
} 


\section{Definition 1. [13]}

For the integer, the Fibonacci sequence is defined by the recurrence relation as

$$
F_{n}=F_{n-1}+F_{n-2}, n \geq 2, F_{0}=0, F_{1}=1
$$

\section{Definition 2. [13]}

For the integer, the Lucas sequence is defined by the recurrence relation as

$$
L_{n}=L_{n-1}+L_{n-2}, n \geq 2, L_{0}=2, L_{1}=1
$$

The generalized Fibonacci sequence $W_{n}=W_{n}(a, b ; p, q)$ is defined as follows:

$$
W_{n}=p W_{n-1}-q W_{n-2}, W_{0}=a, W_{1}=b
$$

where $a, b, p$ and $q$ are arbitrary complex numbers with $q \neq 0$. Since these numbers were first studied by Horadam [4], they are called Horadam numbers. Singh et al. in [11] delineated generalized identities on the relations between Fibonacci and Lucas sequences. Thongmoon in [12] gave identities about the common Factors of Fibonacci and Lucas numbers. Cerin in [2] obtained properties on the factors of summation of consecutive Fibonacci and Lucas numbers.

In 1960 Charles H. King introduced the matrix for classical Fibonacci numbers which is known as $Q$-matrix [9] and $Q$-matrix is given as

$$
Q=\left(\begin{array}{ll}
1 & 1 \\
1 & 0
\end{array}\right)
$$

Cerda [1] studied Horadam sequence (1.3) by matrix methods. Here the author considered two cases of $\left\{W_{n}\right\}$ :

- $\left\{U_{n}\right\}$ is defined by $U_{0}=0$ and $U_{1}=1$

- $\left\{V_{n}\right\}$ is defined by $V_{0}=2$ and $V_{1}=p$

Keskin and Demirturk [6] obtained some new identities for Fibonacci and Lucas numbers by matrix methods. Kilic [7] obtained some summation identities for Fibonacci numbers by matrix methods. In [8] koken and Bozkurt studied and defined a Lucas $Q_{L}$-matrix which is similar to the Fibonacci $Q$-matrix [9] and the $Q_{L}$ matrix is defined as

$$
Q_{L}=\left[\begin{array}{ll}
3 & 1 \\
1 & 2
\end{array}\right] \text { then } Q_{L}^{n}= \begin{cases}5^{\frac{n}{2}}\left[\begin{array}{cc}
F_{n+1} & F_{n} \\
F_{n} & F_{n-1}
\end{array}\right], & \text { for even } n \\
5^{\frac{n-1}{2}}\left[\begin{array}{cc}
L_{n+1} & L_{n} \\
L_{n} & L_{n-1}
\end{array}\right], & \text { for odd } n\end{cases}
$$

where $F_{n}$ and $L_{n}$ are the $n^{\text {th }}$ Fibonacci and Lucas numbers, respectively. Jun and Choi in [5] studied the properties of generalized Fibonacci numbers by matrix methods they defined the generalized Fibonacci 
sequence as

$$
q_{n}=a^{1-\xi(n)} b^{\xi(n)} q_{n-1}+q_{n-2}, n \geq 2, q_{0}=0, q_{1}=1
$$

where $a$ and $b$ are positive real numbers and

$$
\xi(n)=\left\{\begin{array}{l}
0 \text { if } n \text { is even } \\
1 \text { if } n \text { is odd }
\end{array}\right.
$$

In [5] the authors defined the $(2 \times 2)$ matrix $M$ for the above sequence.

$$
M=\left[\begin{array}{cc}
a b & b \\
a & 0
\end{array}\right] \text { and } M^{n}=a^{\frac{n-2+\xi(n)}{2}} b^{\frac{n-\xi(n)}{2}}\left[\begin{array}{cc}
a^{1-\xi(n)} b^{\xi(n)} q_{n+1} & b q_{n} \\
a q_{n} & a^{1-\xi(n)} b^{\xi(n)} q_{n-1}
\end{array}\right]
$$

In addition to this Dasdemir in [3] obtained some identities of Pell, Pell-Lucas and Modified Pell numbers by the matrix methods, in [3] the author defined some two cross two matrices as

$$
N=\left[\begin{array}{ll}
3 & 1 \\
1 & 1
\end{array}\right], \quad R=\left[\begin{array}{ll}
6 & 2 \\
2 & 2
\end{array}\right] \text { and } R=\left[\begin{array}{rr}
2 & 2 \\
2 & -2
\end{array}\right]
$$

\section{Fibonacci-Like sequence and its Matrix Representation}

In this section we define a generalization of Fibonacci sequence which is called Fibonacci-Like sequence also we introduce a $2 \times 2$ matrix representation for Fibonacci-Like sequence.

\section{Definition 3}

For the integers $n \geq 2$ and $p \geq 1$, the Fibonacci-Like sequence is defined by the recurrence relation as

$$
T_{n}=T_{n-1}+T_{n-2}, n \geq 2, T_{0}=p, T_{1}=p
$$

and a $2 \times 2$ matrix representation for Fibonacci-Like sequence and is given by

$$
T=\left[\begin{array}{ll}
\frac{1}{2} & \frac{5}{2} \\
\frac{1}{2} & \frac{1}{2}
\end{array}\right]
$$

\section{Main Results}

In this section we present some main results of this article by using a matrix representation $T$ to FibonacciLike sequence defined in equation (4). 
Lemma 1 If $X$ is a square matrix with $X^{2}=X+I$ then

$$
p X^{n}=T_{n-1} X+T_{n-2} I, n \geq 2
$$

\section{Proof.}

To prove the result we shall use induction on $n$ Let $n=2$, we get

$$
\begin{gathered}
p X^{2}=T_{1} X+T_{0} I \\
p X^{2}=p X+p I \\
X^{2}=X+I
\end{gathered}
$$

Hence the result is true for $n=2$

Assume that the result is true for $n$. Now we show that that the

$$
p X^{n+1}=T_{n} X+T_{n-1} I
$$

Therefore,

$$
\begin{gathered}
T_{n} X+T_{n-1} I=\left(T_{n-1}+T_{n-2}\right) X+T_{n-1} I \\
T_{n} X+T_{n-1} I=(X+I) T_{n-1}+T_{n-2} X \\
T_{n} X+T_{n-1} I=X^{2} T_{n-1}+T_{n-2} X \\
T_{n} X+T_{n-1} I=X^{2} T_{n-1}+T_{n-2} X \\
T_{n} X+T_{n-1} I=X\left(X T_{n-1}+T_{n-2} I\right) \\
T_{n} X+T_{n-1} I=p X\left(X^{n}\right) \\
T_{n} X+T_{n-1} I=p X^{n+1}
\end{gathered}
$$

as required.

Now we show that

$$
p X^{(-n)}=T_{-n-1} X+T_{-n-2} I, n \geq 2
$$

Let 


$$
Y=I-X=-X^{-1}
$$

Therefore,

$$
\begin{gathered}
Y^{2}=(I-X)^{2} \\
Y^{2}=I^{2}-2 X+X^{2} \\
Y^{2}=I-2 X+X+I \\
Y^{2}=I-X+I \\
Y^{2}=Y+I
\end{gathered}
$$

This show that

$$
\begin{gathered}
p Y^{n}=T_{n-1} Y+T_{n-2} I \\
p\left(-X^{-1}\right)^{n}=T_{n-1}(I-X)+T_{n-2} I \\
p\left(-X^{-1}\right)^{n}=T_{n-1}(I-X)+T_{n-2} I \\
p(-1)^{n} X^{-n}=-T_{n-1} X+\left(T_{n-1}+T_{n-2}\right) I \\
p X^{-n}=(-1)^{n+1} T_{n-1} X+(-1)^{n} T_{n} I
\end{gathered}
$$

Since $(-1)^{n+1} T_{n-2}=T_{-n-1}$ and $(-1)^{n+2} T_{n}=T_{-n-2}$ then, we have

$$
p X^{-n}=T_{-n-1} X+T_{-n-2} I
$$

Hence the result.

$$
\begin{gathered}
\text { Theorem } 1 \text { Let } T=\left[\begin{array}{ll}
\frac{1}{2} & \frac{5}{2} \\
\frac{1}{2} & \frac{1}{2}
\end{array}\right] \text { then } \\
T^{n}=\left[\begin{array}{cc}
\frac{L_{n}}{2} & \frac{5 T_{n-1}}{2 p} \\
\frac{T_{n-1}}{2 p} & \frac{L_{n}}{2}
\end{array}\right]
\end{gathered}
$$


Proof. Since $T^{2}=T+I$ then by lemma (3.1), we have

$$
\begin{gathered}
p T^{n}=\left[\begin{array}{cc}
\frac{L_{n}}{2} & \frac{5 T_{n-1}}{2 p} \\
\frac{T_{n-1}}{2 p} & \frac{L_{n}}{2}
\end{array}\right]+\left[\begin{array}{cc}
T_{n-2} & 0 \\
0 & T_{n-2}
\end{array}\right] \\
p T^{n}=\left[\begin{array}{cc}
\frac{T_{n-1}+2 T_{n-2}}{2} & \frac{5 T_{n-1}}{2} \\
\frac{T_{n-1}}{2} & \frac{T_{n-1}+2 T_{n-2}}{2}
\end{array}\right] \\
p T^{n}=\left[\begin{array}{c}
\frac{T_{n-1}+T_{n-2}+T_{n-2}}{2} \\
\frac{5 T_{n-1}}{2} \\
\frac{T_{n-1}}{2}
\end{array}\right] \\
p T^{n}=\left[\begin{array}{cc}
\frac{T_{n-1}+T_{n-2}+T_{n-2}}{2} & \frac{5 T_{n-1}}{2} \\
\frac{T_{n-1}}{2} & \frac{T_{n}+T_{n-2}}{2}
\end{array}\right]
\end{gathered}
$$

Since $T_{n}+T_{n-2}=p L_{n}$ then

$$
\begin{gathered}
p T^{n}=\left[\begin{array}{cc}
\frac{p L_{n}}{2} & \frac{5 T_{n-1}}{2} \\
\frac{T_{n-1}}{2} & \frac{p L_{n}}{2}
\end{array}\right] \\
T^{n}=\left[\begin{array}{ll}
\frac{L_{n}}{2} & \frac{5 T_{n-1}}{2 p} \\
\frac{T_{n-1}}{2 p} & \frac{L_{n}}{2}
\end{array}\right]
\end{gathered}
$$

Hence the result.

Theorem 2 For the positive integer $n$, we have

$$
p^{2} L_{n}^{2}-5 T_{n-1}^{2}=4 p^{2}(-1)^{n}
$$

Proof. Since 


$$
T=\left[\begin{array}{ll}
\frac{1}{2} & \frac{5}{2} \\
\frac{1}{2} & \frac{1}{2}
\end{array}\right] \Rightarrow\left|T^{n}\right|=(-1)^{n}
$$

Again,

$$
T^{n}=\left[\begin{array}{cc}
\frac{L_{n}}{2} & \frac{5 T_{n-1}}{2 p} \\
\frac{T_{n-1}}{2 p} & \frac{L_{n}}{2}
\end{array}\right] \Rightarrow\left|T^{n}\right|=\frac{L_{n}^{2}}{4}-5 \frac{T_{n-1}^{2}}{4 p^{2}}
$$

Hence we conclude that

$$
\frac{L_{n}^{2}}{4}-5 \frac{T_{n-1}^{2}}{4 p^{2}}=(-1)^{n} \Rightarrow p^{2} L_{n}^{2}-5 T_{n-1}^{2}=4 p^{2}(-1)^{n}
$$

Hence the result.

\section{Theorem 3}

$$
\begin{aligned}
& 2 p^{2} L_{n+s}=p^{2} L_{n} L_{s}+5 T_{n-1} T_{s-1}, n \geq 1, s \geq 1 \\
& 2 T_{n+s-1}=T_{n-1} L_{s}+L_{n} T_{s-1}, n \geq 1, s \geq 1
\end{aligned}
$$

\section{Proof.}

$$
\begin{gathered}
T^{n+s}=T^{n} T^{s} \\
T^{n+s}=\left[\begin{array}{cc}
\frac{L_{n}}{2} & \frac{5 T_{n-1}}{2 p} \\
\frac{T_{n-1}}{2 p} & \frac{L_{n}}{2}
\end{array}\right]\left[\begin{array}{cc}
\frac{L_{s}}{2} & \frac{5 T_{s-1}}{2 p} \\
\frac{T_{s-1}}{2 p} & \frac{L_{s}}{2}
\end{array}\right] \\
T^{n+s}=\left[\begin{array}{cc}
\frac{L_{n}}{2} \frac{L_{s}}{2}+5 \frac{T_{n-1}}{2 p} \frac{T_{s-1}}{2 p} & \frac{L_{n}}{2} \frac{5 T_{s-1}}{2 p}+5 \frac{T_{n-1}}{2 p} \frac{L_{s}}{2} \\
\frac{T_{n-1}}{2 p} \frac{L_{s}}{2}+\frac{L_{n}}{2} \frac{T_{s-1}}{2 p} & 5 \frac{T_{n-1} \frac{T_{s-1}}{2 p}+\frac{L_{n}}{2} \frac{L_{s}}{2}}{2 p}
\end{array}\right] \\
T^{n+s}=\left[\begin{array}{cc}
\frac{p^{2} L_{n} L_{s}+5 T_{n-1} T_{s-1}}{4 p^{2}} & \frac{5\left(L_{n} T_{s-1}+T_{n-1} L_{s}\right)}{4 p} \\
\frac{T_{n-1} L_{s}+L_{n} T_{s-1}}{4 p} & \frac{5 T_{n-1} T_{s-1}+p^{2} L_{n} L_{s}}{4 p^{2}}
\end{array}\right]
\end{gathered}
$$


But,

$$
T^{n+s}=\left[\begin{array}{ll}
\frac{L_{n+s}}{2} & \frac{5 T_{n+s-1}}{2 p} \\
\frac{T_{n+s-1}}{2 p} & \frac{L_{n+s}}{2}
\end{array}\right]
$$

Gives,

$$
\begin{gathered}
\frac{L_{n+s}}{2}=\frac{p^{2} L_{n} L_{s}+5 T_{n-1} T_{s-1}}{4 p^{2}} \\
2 p^{2} L_{n+s}=p^{2} L_{n} L_{s}+5 T_{n-1} T_{s-1}
\end{gathered}
$$

and,

$$
\begin{aligned}
& \frac{T_{n+s-1}}{2 p}=\frac{T_{n-1} L_{s}+L_{n} T_{s-1}}{4 p} \\
& 2 T_{n+s-1}=T_{n-1} L_{s}+L_{n} T_{s-1}
\end{aligned}
$$

Hence the theorem.

\section{Theorem 4}

$$
\begin{aligned}
& 2 p^{2}(-1)^{s} L_{n-s}=p^{2} L_{n} L_{s}-5 T_{n-1} T_{s-1}, n \geq 1, s \geq 1 \\
& 2(-1)^{s} T_{n-s-1}=T_{n-1} L_{s}-L_{n} T_{s-1}, 1 \leq s \leq n
\end{aligned}
$$

Proof. Since

$$
T^{s}=\left[\begin{array}{cc}
\frac{L_{s}}{2} & \frac{5 T_{s-1}}{2 p} \\
\frac{T_{s-1}}{2 p} & \frac{L_{s}}{2}
\end{array}\right] \Rightarrow T^{-s}=\left[\begin{array}{cc}
\frac{L_{s}}{2} & \frac{5 T_{s-1}}{2 p} \\
\frac{T_{s-1}}{2 p} & \frac{L_{s}}{2}
\end{array}\right]^{-1}
$$

Then by theorem (2), we have

$$
T^{-s}=\frac{1}{(-1)^{s}}\left[\begin{array}{cc}
\frac{L_{s}}{2} & -\frac{5 T_{s-1}}{2 p} \\
-\frac{T_{s-1}}{2 p} & \frac{L_{s}}{2}
\end{array}\right]
$$




$$
\begin{gathered}
T^{n-s}=T^{n} T^{-s} \Rightarrow T^{n-s}=\left[\begin{array}{cc}
\frac{L_{n}}{2} & \frac{5 T_{n-1}}{2 p} \\
\frac{T_{n-1}}{2 p} & \frac{L_{n}}{2}
\end{array}\right] \frac{1}{(-1)^{s}}\left[\begin{array}{cc}
\frac{L_{s}}{2} & -\frac{5 T_{s-1}}{2 p} \\
-\frac{T_{s-1}}{2 p} & \frac{L_{s}}{2}
\end{array}\right] \\
T^{n-s}=\frac{1}{(-1)^{s}}\left[\begin{array}{cc}
\frac{L_{n}}{2} & \frac{5 T_{n-1}}{2 p} \\
\frac{T_{n-1}}{2 p} & \frac{L_{n}}{2}
\end{array}\right]\left[\begin{array}{cc}
\frac{L_{s}}{2} & -\frac{5 T_{s-1}}{2 p} \\
-\frac{T_{s-1}}{2 p} & \frac{L_{s}}{2}
\end{array}\right] \\
T^{n-s}=(-1)^{-s}\left[\begin{array}{cc}
\frac{p^{2} L_{n} L_{s}-5 T_{n-1} T_{s-1}}{4 p^{2}} & \frac{5\left(T_{n-1} L_{s}-L_{n} T_{s-1}\right)}{4 p} \\
\frac{T_{n-1} L_{s}-L_{n} T_{s-1}}{4 p} & \frac{p^{2} L_{n} L_{s}-5 T_{n-1} T_{s-1}}{4 p^{2}}
\end{array}\right]
\end{gathered}
$$

But,

$$
T^{n-s}=\left[\begin{array}{ll}
\frac{L_{n-s}}{2} & \frac{5 T_{n-s-1}}{2 p} \\
\frac{T_{n-s-1}}{2 p} & \frac{L_{n-s}}{2}
\end{array}\right]
$$

Then,

$$
\begin{gathered}
\frac{L_{n-s}}{2}=(-1)^{-s} \frac{p^{2} L_{n} L_{s}-5 T_{n-1} T_{s-1}}{4 p^{2}} \\
2 p^{2}(-1)^{s} L_{n-s}=p^{2} L_{n} L_{s}-5 T_{n-1} T_{s-1}
\end{gathered}
$$

and,

$$
\begin{aligned}
& \frac{T_{n-s-1}}{2 p}=(-1)^{-s} \frac{T_{n-1} L_{s}-L_{n} T_{s-1}}{4 p} \\
& 2(-1)^{s} T_{n-s-1}=T_{n-1} L_{s}-L_{n} T_{s-1}
\end{aligned}
$$

\section{Theorem 5}

$$
\begin{aligned}
& L_{n} L_{s}=L_{n+s}+(-1)^{s} L_{n-s}, 1 \leq s \leq n \\
& 2(-1)^{s} T_{n-s-1}=\frac{T_{n+s-1}+(-1)^{s} T_{n-s-1}}{T_{n-1}}, 1 \leq s \leq n
\end{aligned}
$$


Proof. Since

$$
T^{n+s}=\left[\begin{array}{ll}
\frac{L_{n+s}}{2} & \frac{5 T_{n+s-1}}{2 p} \\
\frac{T_{n+s-1}}{2 p} & \frac{L_{n+s}}{2}
\end{array}\right] \text { and }(-1)^{s} T^{n-s}=\left[\begin{array}{ll}
(-1)^{s} \frac{L_{n+s}}{2} & (-1)^{s} \frac{5 T_{n+s-1}}{2 p} \\
(-1)^{s} \frac{T_{n+s-1}}{2 p} & (-1)^{s} \frac{L_{n+s}}{2}
\end{array}\right]
$$

Therefore,

$$
T^{n+s}+(-1)^{s} T^{n-s}=\left[\begin{array}{ll}
\frac{L_{n+s}+(-1)^{s} L_{n-s}}{2} & \frac{5\left[T_{n+s-1}+(-1)^{s} T_{n-s-1}\right]}{2 p} \\
\frac{T_{n+s-1}+(-1)^{s} T_{n-s-1}}{2 p} & \frac{L_{n+s}+(-1)^{s} L_{n-s}}{2}
\end{array}\right]
$$

On the other hand,

$$
\begin{aligned}
& T^{n+s}+(-1)^{s} T^{n-s}=T^{n} T^{s}+(-1)^{s} T^{n} T^{-s} \\
& T^{n+s}+(-1)^{s} T^{n-s}=T^{n}\left[T^{s}+(-1)^{s} T^{-s}\right\rfloor \\
& T^{n+s}+(-1)^{s} T^{n-s}=T^{n}\left[\left(\begin{array}{cc}
\frac{L_{s}}{2} & \frac{5 T_{s-1}}{2 p} \\
\frac{T_{s-1}}{2 p} & \frac{L_{s}}{2}
\end{array}\right)+\frac{(-1)^{s}}{(-1)^{s}}\left(\begin{array}{cc}
\frac{L_{s}}{2} & -\frac{5 T_{s-1}}{2 p} \\
-\frac{T_{s-1}}{2 p} & \frac{L_{s}}{2}
\end{array}\right)\right] \\
& T^{n+s}+(-1)^{s} T^{n-s}=\left[\begin{array}{cc}
\frac{L_{n}}{2} & \frac{5 T_{n-1}}{2 p} \\
\frac{T_{n-1}}{2 p} & \frac{L_{n}}{2}
\end{array}\right]\left[\begin{array}{cc}
L_{s} & 0 \\
0 & L_{s}
\end{array}\right] \\
& T^{n+s}+(-1)^{s} T^{n-s}=\left[\begin{array}{ll}
\frac{L_{n} L_{s}}{2} & \frac{5 T_{n-1} L_{s}}{2 p} \\
\frac{T_{n-1} L_{s}}{2 p} & \frac{L_{n} L_{s}}{2}
\end{array}\right]
\end{aligned}
$$

Hence,

$$
\begin{aligned}
& \frac{L_{n+s}+(-1)^{s} L_{n-s}}{2}=\frac{L_{n} L_{s}}{2} \\
& L_{n} L_{s}=L_{n+s}+(-1)^{s} L_{n-s}
\end{aligned}
$$


and

$$
\begin{gathered}
\frac{T_{n+s-1}+(-1)^{s} T_{n-s-1}}{2 p}=\frac{T_{n-1} L_{s}}{2 p} \\
T_{n-1} L_{s}=T_{n+s-1}+(-1)^{s} T_{n-s-1} \\
L_{s}=\frac{T_{n+s-1}+(-1)^{s} T_{n-s-1}}{T_{n-1}}
\end{gathered}
$$

\section{Theorem 6}

$$
\begin{aligned}
& 4 p^{2} L_{x+y+z}=p^{2} L_{x} L_{y} L_{z}+5\left(L_{x} T_{y-1} T_{z-1}+T_{x-1} L_{y} T_{z-1}+T_{x-1} T_{y-1} L_{z}\right), x \geq 1, y \geq 1, z \geq 1 \\
& 4 p^{2} T_{x+y+z-1}=p^{2}\left(L_{x} L_{y} T_{z-1}+T_{x-1} L_{y} L_{z}+L_{x} L_{y-1} L_{z}\right)+5 T_{x-1} T_{y-1} T_{z-1}
\end{aligned}
$$

where $x \geq 1, y \geq 1, z \geq 1$ and $x+y+z \geq 1$

Proof. By the definition of the matrix $T^{n}$, we have

$$
T^{x+y+z}=\left[\begin{array}{ll}
\frac{L_{x+y+z}}{2} & \frac{5 T_{x+y+z-1}}{2 p} \\
\frac{T_{x+y+z-1}}{2 p} & \frac{L_{x+y+z}}{2}
\end{array}\right]
$$

Again,

$$
\begin{aligned}
T^{x+y+z} & =T^{x+y} T^{z} \Rightarrow T^{x+y+z}=\left[\begin{array}{cc}
\frac{L_{x+y}}{2} & \frac{5 T_{x+y-1}}{2 p} \\
\frac{T_{x+y-1}}{2 p} & \frac{L_{x+y}}{2}
\end{array}\right]\left[\begin{array}{cc}
\frac{L_{z}}{2} & \frac{5 T_{z-1}}{2 p} \\
\frac{T_{z-1}}{2 p} & \frac{L_{z}}{2}
\end{array}\right] \\
T^{x+y+z} & =\left[\begin{array}{ll}
\frac{L_{x+y}}{2} \frac{L_{z}}{2}+5 \frac{T_{x+y-1}}{2 p} \frac{T_{z-1}}{2 p} & \frac{L_{x+y}}{2} \frac{5 T_{z-1}}{2 p}+5 \frac{T_{x+y-1}}{2 p} \frac{L_{z}}{2} \\
\frac{T_{x+y-1} \frac{L_{z}}{2}+\frac{L_{x+y}}{2} \frac{T_{z-1}}{2 p}}{2 p} & \frac{5 \frac{T_{z-1}}{2 p} \frac{T_{x+y-1}}{2 p}+\frac{L_{x+y}}{2} \frac{L_{z}}{2}}{2}
\end{array}\right] \\
T^{x+y+z} & =\left[\begin{array}{cc}
\frac{p^{2} L_{x+y} L_{z}+5 T_{x+y-1} T_{z-1}}{4 p^{2}} & \frac{5\left(L_{x+y} T_{z-1}+T_{x+y-1} L_{z}\right)}{4 p} \\
\frac{T_{x+y-1} L_{z}+L_{x+y} T_{z-1}}{4 p} & \frac{5 T_{x+y-1} T_{z-1}+p^{2} L_{x+y} L_{z}}{4 p^{2}}
\end{array}\right]
\end{aligned}
$$


Equating corresponding terms of the the two matrices, we get

$$
\begin{gathered}
\frac{L_{x+y+z}}{2}=\frac{p^{2} L_{x+y} L_{s}+5 T_{x+y-1} T_{z-1}}{4 p^{2}} \\
4 p^{2} L_{x+y+z}=\left(2 p^{2} L_{x+y}\right) L_{z}+5\left(2 T_{x+y-1}\right) T_{z-1}
\end{gathered}
$$

Using theorem (3), we have

$$
\begin{gathered}
4 p^{2} L_{x+y+z}=\left(p^{2} L_{x} L_{y}+5 T_{x-1} T_{y-1}\right) L_{z}+5\left(T_{x-1} L_{y}+L_{x} T_{y-1}\right) T_{z-1} \\
4 p^{2} L_{x+y+z}=p^{2} L_{x} L_{y} L_{z}+5 T_{x-1} T_{y-1} L_{z}+5 T_{x-1} L_{y} T_{z-1}+5 L_{x} T_{y-1} T_{z-1} \\
4 p^{2} L_{x+y+z}=p^{2} L_{x} L_{y} L_{z}+5\left(L_{x} T_{y-1} T_{z-1}+T_{x-1} L_{y} T_{z-1}+T_{x-1} T_{y-1} L_{z}\right)
\end{gathered}
$$

and,

$$
\begin{gathered}
\frac{T_{x+y+z-1}}{2 p}=\frac{T_{x+y-1-1} L_{z}+L_{x+y} T_{z-1}}{4 p} \\
4 p T_{x+y+z-1}=p\left(2 T_{x+y-1}\right) L_{z}+\left(2 p L_{x+y}\right) T_{z-1} \\
4 p^{2} T_{x+y+z-1}=p^{2}\left(2 T_{x+y-1}\right) L_{z}+\left(2 p^{2} L_{x+y}\right) T_{z-1}
\end{gathered}
$$

Using theorem (3), we have

$$
\begin{gathered}
4 p^{2} T_{x+y+z-1}=p^{2}\left(T_{x-1} L_{y}+L_{x} T_{y-1}\right) L_{z}+\left(p^{2} L_{x} L_{y}+5 T_{x-1} T_{y-1}\right) T_{z-1} \\
4 p^{2} T_{x+y+z-1}=p^{2} T_{x-1} L_{y} L_{z}+p^{2} L_{x} T_{y-1} L_{z}+p^{2} L_{x} L_{y} T_{z-1}+5 T_{x-1} T_{y-1} T_{z-1} \\
4 p^{2} T_{x+y+z-1}=p^{2}\left(L_{x} L_{y} T_{z-1}+T_{x-1} L_{y} L_{z}+L_{x} L_{y-1} L_{z}\right)+5 T_{x-1} T_{y-1} T_{z-1}
\end{gathered}
$$

Hence the theorem.

\section{Theorem 7}

$$
\begin{gathered}
L_{y}=\frac{(-1)^{-x}}{L_{z-x}} p^{2} L_{x+y} L_{z}-5 T_{x-1} T_{y+z-1}, 1 \leq x \leq z, y \geq 1 \quad y+z \geq 1 \\
T_{y-1}=\frac{p^{2}(-1)^{-x}}{L_{z-x}} L_{x} T_{y+z-1}-T_{z-1} L_{x+y}, 1 \leq x \leq z, y \geq 1 \quad y+z \geq 1
\end{gathered}
$$


Proof. Let us consider a product

$$
\begin{gathered}
B_{1}=\left[\begin{array}{cc}
\frac{L_{x}}{2} & \frac{5 T_{x-1}}{2 p} \\
\frac{T_{z-1}}{2 p} & \frac{L_{z}}{2}
\end{array}\right]\left[\begin{array}{c}
L_{y} \\
\frac{T_{y-1}}{p}
\end{array}\right]=\left[\begin{array}{c}
\frac{L_{x}}{2} L_{y}+\frac{5 T_{x-1}}{2 p} \frac{T_{y-1}}{p} \\
\frac{T_{z-1}}{2 p} L_{y}+\frac{L_{z}}{2} \frac{T_{y-1}}{p}
\end{array}\right] \\
B_{1}=\left[\begin{array}{c}
\frac{p^{2} L_{x} L_{y}+5 T_{x-1} T_{y-1}}{2 p^{2}} \\
\frac{T_{z-1} L_{y}+L_{z} T_{y-1}}{2 p}
\end{array}\right]
\end{gathered}
$$

From theorem (3), we have

$$
\begin{gathered}
B_{1}=\left[\begin{array}{c}
\frac{2 p^{2} L_{x+y}}{2 p^{2}} \\
\frac{2 T_{y+z-1}}{2 p}
\end{array}\right]=\left[\begin{array}{c}
L_{x+y} \\
\frac{T_{y+z-1}}{p}
\end{array}\right] \text { then } \\
B_{1}=\left[\begin{array}{cc}
\frac{L_{x}}{2} & \frac{5 T_{x-1}}{2 p} \\
\frac{T_{z-1}}{2 p} & \frac{L_{z}}{2}
\end{array}\right]\left[\begin{array}{c}
L_{y} \\
\frac{T_{y-1}}{p}
\end{array}\right]=\left[\begin{array}{c}
L_{x+y} \\
\frac{T_{y+z-1}}{p}
\end{array}\right]
\end{gathered}
$$

Let

$$
B_{2}=\left|\begin{array}{cc}
\frac{L_{x}}{2} & \frac{5 T_{x-1}}{2 p} \\
\frac{T_{z-1}}{2 p} & \frac{L_{z}}{2}
\end{array}\right|=\frac{L_{x} L_{z}}{4}-5 \frac{T_{x-1} T_{z-1}}{4 p^{2}} \Rightarrow B_{2}=\frac{p^{2} L_{x} L_{z}-5 T_{x-1} T_{z-1}}{4 p^{2}}
$$

Again by theorem (4), we have

$$
B_{2}=\frac{2(-1)^{x} L_{z-x}}{4 p^{2}}=\frac{(-1)^{x} L_{z-x}}{2 p^{2}} \neq 0, \text { for } z \neq x
$$

Therefore,

$$
\left[\begin{array}{c}
L_{y} \\
\frac{T_{y-1}}{p}
\end{array}\right]=\left[\begin{array}{cc}
\frac{L_{x}}{2} & \frac{5 T_{x-1}}{2 p} \\
\frac{T_{z-1}}{2 p} & \frac{L_{z}}{2}
\end{array}\right]^{-1}\left[\begin{array}{c}
L_{x+y} \\
\frac{T_{y+z-1}}{p}
\end{array}\right]
$$




$$
\begin{aligned}
& {\left[\begin{array}{c}
L_{y} \\
\frac{T_{y-1}}{p}
\end{array}\right]=\frac{2 p^{2}(-1)^{-x}}{L_{z-x}}\left[\begin{array}{ll}
\frac{L_{z}}{2} & \frac{-5 T_{x-1}}{2 p} \\
\frac{-T_{z-1}}{2 p} & \frac{L_{x}}{2}
\end{array}\right]\left[\begin{array}{c}
L_{x+y} \\
T_{y+z-1} \\
p
\end{array}\right]} \\
& {\left[\begin{array}{c}
L_{y} \\
\frac{T_{y-1}}{p}
\end{array}\right]=\frac{2 p^{2}(-1)^{-x}}{L_{z-x}}\left[\begin{array}{l}
\frac{L_{z}}{2} L_{x+y} \frac{-5 T_{x-1}}{2 p} \frac{T_{y+z-1}}{p} \\
\frac{-T_{z-1}}{2 p} L_{x+y}+\frac{L_{x}}{2} \frac{T_{y+z-1}}{p}
\end{array}\right]} \\
& {\left[\begin{array}{c}
L_{y} \\
\frac{T_{y-1}}{p}
\end{array}\right]=\frac{2 p^{2}(-1)^{-x}}{L_{z-x}}\left[\begin{array}{l}
\frac{p^{2} L_{z} L_{x+y}-5 T_{x-1} T_{y+z-1}}{2 p^{2}} \\
\frac{L_{x} T_{y+z-1}-T_{z-1} L_{x+y}}{2 p}
\end{array}\right]}
\end{aligned}
$$

Therefore,

$$
\begin{gathered}
L_{y}=\frac{(-1)^{-x}}{L_{z-x}} p^{2} L_{z} L_{x+y}-5 T_{x-1} T_{y+z-1} \\
\frac{T_{y-1}}{p}=\frac{2 p(-1)^{-x}}{L_{z-x}} L_{x} T_{y+z-1}-T_{z-1} L_{x+y} \\
T_{y-1}=\frac{2 p^{2}(-1)^{-x}}{L_{z-x}} L_{x} T_{y+z-1}-T_{z-1} L_{x+y}
\end{gathered}
$$

\section{Theorem 8}

$$
\begin{aligned}
& L_{y}=\frac{(-1)^{-x}}{T_{z-x-1}} L_{x+y} T_{z-1}-T_{x-1} L_{y+z}, 1 \leq x \leq z, y \geq 1 \\
& T_{y-1}=\frac{p^{2}(-1)^{-x}}{5 T_{z-x-1}} L_{x} L_{y+z}-L_{z} L_{x+y}, 1 \leq x \leq z, y \geq 1
\end{aligned}
$$

Proof. Let us consider a product

$$
B_{3}=\left[\begin{array}{cc}
\frac{L_{x}}{2} & \frac{5 T_{x-1}}{2 p} \\
\frac{L_{z}}{2} & 5 \frac{T_{z-1}}{2 p}
\end{array}\right]\left[\begin{array}{c}
L_{y} \\
\frac{T_{y-1}}{p}
\end{array}\right]=\left[\begin{array}{l}
\frac{L_{x}}{2} L_{y}+\frac{5 T_{x-1}}{2 p} \frac{T_{y-1}}{p} \\
\frac{L_{z}}{2} L_{y}+5 \frac{T_{z-1}}{2 p} \frac{T_{y-1}}{p}
\end{array}\right]
$$




$$
B_{3}=\left[\begin{array}{l}
\frac{p^{2} L_{x} L_{y}+5 T_{x-1} T_{y-1}}{2 p^{2}} \\
\frac{p^{2} L_{z} L_{y}+5 T_{z-1} T_{y-1}}{2 p^{2}}
\end{array}\right]
$$

From theorem (3), we have

$$
\begin{gathered}
B_{3}=\left[\begin{array}{c}
\frac{2 p^{2} L_{x+y}}{2 p^{2}} \\
\frac{2 p^{2} L_{y+z}}{2 p^{2}}
\end{array}\right]=\left[\begin{array}{c}
L_{x+y} \\
L_{y+z}
\end{array}\right] \text { then } \\
B_{3}=\left[\begin{array}{cc}
\frac{L_{x}}{2} & \frac{5 T_{x-1}}{2 p} \\
\frac{L_{z}}{2} & 5 \frac{T_{z-1}}{2 p}
\end{array}\right]\left[\begin{array}{c}
L_{y} \\
\frac{T_{y-1}}{p}
\end{array}\right]=\left[\begin{array}{l}
L_{x+y} \\
L_{y+z}
\end{array}\right]
\end{gathered}
$$

Let

$$
B_{4}=\left[\begin{array}{cc}
\frac{L_{x}}{2} & \frac{5 T_{x-1}}{2 p} \\
\frac{L_{z}}{2} & 5 \frac{T_{z-1}}{2 p}
\end{array}\right]=\frac{L_{x}}{2} 5 \frac{T_{z-1}}{2 p}-\frac{L_{z}}{2} 5 \frac{T_{x-1}}{2 p} \Rightarrow B_{4}=\frac{5\left(L_{x} T_{z-1}-L_{z} T_{x-1}\right)}{4 p}
$$

Again by theorem (4), we have

$$
B_{4}=\frac{5}{4 p} \frac{\left[2(-1)^{x} T_{z-x-1}\right]}{4 p}=\frac{5(-1)^{x} T_{z-x-1}}{2 p} \neq 0
$$

Therefore,

$$
\begin{gathered}
{\left[\begin{array}{c}
L_{y} \\
\frac{T_{y-1}}{p}
\end{array}\right]=\left[\begin{array}{cc}
\frac{L_{x}}{2} & \frac{5 T_{x-1}}{2 p} \\
\frac{L_{z}}{2} & 5 \frac{T_{z-1}}{2 p}
\end{array}\right]^{-1}\left[\begin{array}{c}
L_{x+y} \\
L_{y+z}
\end{array}\right]} \\
{\left[\begin{array}{c}
L_{y} \\
\frac{T_{y-1}}{p}
\end{array}\right]=\frac{2 p(-1)^{-x}}{5 T_{z-x-1}}\left[\begin{array}{cc}
5 \frac{T_{z-1}}{2 p} & -\frac{5 T_{x-1}}{2 p} \\
-\frac{L_{z}}{2} & \frac{L_{x}}{2}
\end{array}\right]\left[\begin{array}{l}
L_{x+y} \\
L_{y+z}
\end{array}\right]}
\end{gathered}
$$




$$
\begin{aligned}
& {\left[\begin{array}{c}
L_{y} \\
\frac{T_{y-1}}{p}
\end{array}\right]=\frac{2 p(-1)^{-x}}{5 T_{z-x-1}}\left[\begin{array}{c}
\frac{5 T_{z-1}}{2 p} L_{x+y}-5 \frac{T_{x-1}}{2 p} L_{y+z} \\
-\frac{L_{z}}{2} L_{x+y}+\frac{L_{x}}{2} L_{y+z}
\end{array}\right]} \\
& {\left[\begin{array}{c}
L_{y} \\
\frac{T_{y-1}}{p}
\end{array}\right]=\frac{2 p(-1)^{-x}}{5 T_{z-x-1}}\left[\begin{array}{c}
\frac{5\left(T_{z-1} L_{x+y}-T_{x-1} L_{y+z}\right)}{2 p} \\
\frac{L_{x} L_{y+z}-L_{z} L_{x+y}}{2}
\end{array}\right]}
\end{aligned}
$$

Therefore,

$$
\begin{gathered}
L_{y}=\frac{2 p(-1)^{-x}}{5 T_{z-x-1}} \frac{5\left(T_{z-1} L_{x+y}-T_{x-1} L_{y+z}\right)}{2 p} \\
L_{y}=\frac{(-1)^{-x}}{T_{z-x-1}} T_{z-1} L_{x+y}-T_{x-1} L_{y+z}
\end{gathered}
$$

and

$$
\begin{gathered}
\frac{T_{y-1}}{p}=\frac{2 p(-1)^{-x}}{5 T_{z-x-1}} \frac{L_{x} L_{y+z}-L_{z} L_{x+y}}{2} \\
\frac{T_{y-1}}{p}=\frac{p(-1)^{-x}}{5 T_{z-x-1}} L_{x} L_{y+z}-L_{z} L_{x+y} \\
T_{y-1}=\frac{p^{2}(-1)^{-x}}{5 T_{z-x-1}} L_{x} L_{y+z}-L_{z} L_{x+y}
\end{gathered}
$$

Hence the result

\section{Conclusion}

In this article we presented a generalized Fibonacci sequence called Fibonacci-Like sequence and after that some relations have been obtained between Lucas sequence and Fibonacci-Like sequence by matrix methods.

\section{References}

[1] Cerda, G., "Matrix Methods in Horadam Sequences”, Boletín de Matemáticas, 19(2), 2012, pp.97-106.

[2] Čerin, Z., "On Factors of Sums of Consecutive Fibonacci and Lucas Numbers", Annales Mathematicae et Informaticae, 41, 2013, pp. 19-25.

[3] Dasdemir, A., "On the Pell, Pell-Lucas and Modified Pell Numbers by Matrix Method", Applied 
Mathematical Sciences, 5(64), 2011, pp.3173-3181.

[4] Horadam, A. F., "Basic Properties of a Certain Generalized Sequence of Numbers", The Fibonacci Quarterly, 3(3), 1965, pp.161-176.

[5] Jun, S. P. and Choi, K. H., "Some Properties of the Generalized Fibonacci Sequence $\left\{q_{n}\right\}$ by Matrix Methods Methods", The Korean Journal of Mathematics, 24(4), 2016, pp.681-691.

[6] Keskin, R. and Demirtürk, B., "Some New Fibonacci and Lucas Identities by Matrix Methods", International Journal of Mathematical Education in Science and Technology, 41(3), 2010, pp.379-387.

[7] Kilic, E., "Sums of Generalized Fibonacci Numbers by Matrix Methods", Ars Combinatoria, 84, 2007, pp.23-32.

[8] Koken, F. and Bozkurt, D., "On Lucas Numbers by the Matrix Method", Hacettepe Journal of Mathematics and Statistics, 39(4), 2010, pp.471-475.

[9] Koshy, T., Fibonacci and Lucas Numbers with Applications, John Wiley \& Sons, 2011.

[10] Posamentier, A. S., The Fabulous Fibonacci Numbers, Prometheus Books, 2007.

[11] Singh, B., Bhadouria, P. and Sikhwal, O., "Generalized Identities Involving Common Factors of Fibonacci and Lucas Numbers", International Journal of Algebra, 5(13), 2011, pp.637-645.

[12] Thongmoon, M., 2009, "Identities for the Common Factors of Fibonacci and Lucas Numbers", International Mathematical Forum, 4(7), 2009, pp. 303-308.

[13] Vajda, S., Fibonacci and Lucas Numbers and the Golden Section: Theory and Applications, Courier Corporation, 1989.

\section{Authors' Profiles}

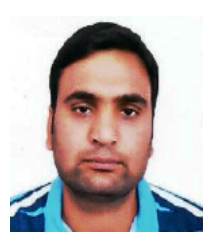

Arfat Ahmad Wani, has completed post graduation in mathematics in 2013 from University of Kashmir. After that in 2014 he enrolled as a Ph. D research scholar in School of Studies in Mathematics Vikram University Ujjain, India and currently he is carrying a research work in Number Theory on Fibonacci Numbers.

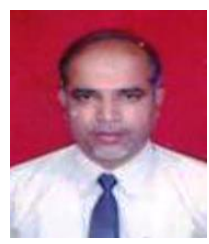

V. H. Badshah, Professor and Head in School of Studies in Mathematics Vikram University Ujjain, India. He is M.sc and Ph.d from also School of Studies in Mathematics Vikram University Ujjain, India. His research specialization in Number Theory as well as in Functional Analysis. He published about 200 papers in international and national reputed journals.

How to cite this paper: Arfat Ahmad Wani, V. H. Badshah,"On the Relations between Lucas Sequence and Fibonacci-like Sequence by Matrix Methods", International Journal of Mathematical Sciences and Computing(IJMSC), Vol.3, No.4, pp.20-36, 2017.DOI: 10.5815/ijmsc.2017.04.03 